\title{
Spontaneous activity in peripheral diaphragmatic lymphatic loops
}

Andrea Moriondo, Eleonora Solari, Cristiana Marcozzi and Daniela Negrini

Am J Physiol Heart Circ Physiol 305:H987-H995, 2013. First published 26 July 2013;

doi:10.1152/ajpheart.00418.2013

You might find this additional info useful...

This article cites 23 articles, 13 of which can be accessed free at:

/content/305/7/H987.full.html\#ref-list-1

Updated information and services including high resolution figures, can be found at:

/content/305/7/H987.full.html

Additional material and information about $\boldsymbol{A J P}$ - Heart and Circulatory Physiology can be found at: http://www.the-aps.org/publications/ajpheart

This information is current as of October 3, 2013.

\footnotetext{
AJP - Heart and Circulatory Physiology publishes original investigations on the physiology of the heart, blood vessels, and lymphatics, including experimental and theoretical studies of cardiovascular function at all levels of organization ranging from the intact animal to the cellular, subcellular, and molecular levels. It is published 12 times a year (monthly) by the American

Physiological Society, 9650 Rockville Pike, Bethesda MD 20814-3991. Copyright @ 2013 by the American Physiological Society. ISSN: 0363-6135, ESSN: 1522-1539. Visit our website at http://www.the-aps.org/.
} 


\title{
Spontaneous activity in peripheral diaphragmatic lymphatic loops
}

\author{
Andrea Moriondo, Eleonora Solari, Cristiana Marcozzi, and Daniela Negrini \\ Department of Surgical and Morphological Sciences, University of Insubria, Varese, Italy
}

Submitted 20 May 2013; accepted in final form 23 July 2013

\begin{abstract}
Moriondo A, Solari E, Marcozzi C, Negrini D. Spontaneous activity in peripheral diaphragmatic lymphatic loops. Am J Physiol Heart Circ Physiol 305: H987-H995, 2013. First published July 26, 2013; doi:10.1152/ajpheart.00418.2013. - The spontaneous contractility of FITC-dextran-filled lymphatics at the periphery of the pleural diaphragm was documented for the first time "in vivo" in anesthetized Wistar rats. We found that lymphatic segments could be divided into four phenotypes: 1) active, displaying rhythmic spontaneous contractions (51.8\% of 197 analyzed sites); 2) stretch-activated, whose contraction was triggered by passive distension of the vessel lumen $(4.1 \%)$; 3) passive, which displayed a completely passive distension $(4.5 \%)$; and 4$)$ inert, whose diameter never changed over time $(39.6 \%)$. Smooth muscle actin was detected by immunofluorescence and confocal microscopy in the vessel walls of active but also of inert sites, albeit with a very different structure within the vessel wall. Indeed, while in active segments, actin was arranged in a dense mesh completely surrounding the lumen, in inert segments actin decorated the vessels wall in sparse longitudinal strips. When located nearby along the same lymphatic loop, active, stretch-activated, and passive sites were always recruited in temporal sequence starting from the active contraction. The time delay was $\sim 0.35 \mathrm{~s}$ between active and stretch-activated and $0.54 \mathrm{~s}$ between stretch-activated and passive segments, promoting a uniform lymph flux of $\sim 150 / 200 \mathrm{pl} / \mathrm{min}$. We conclude that, unlike more central diaphragmatic lymphatic vessels, loops located at the extreme diaphragmatic periphery do require an intrinsic pumping mechanism to propel lymph centripetally, and that such an active lymph propulsion is attained by means of a complex interplay among sites whose properties differ but are indeed able to organize lymph flux in an ordered fashion.
\end{abstract}

diaphragmatic lymphatic network; initial lymphatics; intrinsic lymphatic mechanism

THE DIAPHRAGMATIC LYMPHATIC network is a complex vascular system arranged both over the pleural and peritoneal sides of the diaphragm $(3,18)$. Both linear lymphatics and vessels are arranged in complex loops that run parallel to the diaphragmatic surface, forming a superficial submesothelial network. Transverse ducts depart from these vessels and run perpendicular to the diaphragmatic surface through the skeletal muscle fibers to reach larger collecting lymphatics, located in the center of the diaphragmatic thickness and supposed to propel the lymph away from the diaphragm (7). Throughout the body tissues, lymph formation and propulsion have been found to rely on two different mechanisms: one intrinsic, due to the rhythmic spontaneous contraction of the smooth muscle cells of the lymphatic vessel wall (12), and the other extrinsic, associated with tissue displacements and depending on the mechanical stresses arising in the tissue surrounding the lymphatics (15). Diaphragmatic lymphatic function has been extensively studied by means of fluorescence in vivo imaging and micropuncture technique in loops and linear vessels that are

Address for reprint requests and other correspondence: D. Negrini, DSCM - Via J.H. Dunant, 5, I-21100 Varese, Italy (e-mail: daniela.negrini@uninsubria.it). located in the muscular region just below the mesothelial layer. In this superficial network of the diaphragm, both cardiac and respiratory activities support the extrinsic mechanism $(8,11$, 17), while no significant evidence of the intrinsic pumping mechanism has been observed. Indeed, the submesothelial lymphatic vessels studied so far are initial lymphatics, since they are devoid of any muscular layer in their walls; in these vessels, the cyclic contractions/relaxations of the diaphragm during respiratory activity and local tissue displacements due to the propagation of cardiogenic pressure waves provide an effective extrinsic pumping mechanism apparently able to drain fluid from the pleural and peritoneal cavity and from the surrounding muscular interstitium (17). In these previous studies, lymphatics located at the conjunction between the diaphragm and the abdominal wall have never been investigated in deep, given their rather hidden, extreme peripheral location just beneath the costal margin facing the apposition zone.

In the present work, we show for the first time that, at variance with what was observed for vessels located in the diaphragmatic muscular portion, lymphatic loops at the very far periphery of the diaphragm do possess an intrinsic pumping mechanism. Not only are these vessels capable of spontaneous contraction, but their contractile machinery is unevenly distributed within a loop. However, despite the variability of the segmental function, the loops seems to behave as a well-coordinated unit, providing an unidirectional and almost steady lymph flux toward the more central and deep regions of the diaphragmatic lymphatic network.

\section{METHODS}

Surgical procedures and in vivo vessel activity recording. Experiments were performed on 11 adult Wistar rats (mean body weight $327 \pm 33 \mathrm{~g}$ ) anesthetized with an intraperitoneal injection of $75 \mathrm{mg} / \mathrm{kg}$ body wt ketamine and $0.5 \mathrm{mg} / \mathrm{kg}$ body wt medetomidine cocktail in saline solution. Successive boluses were administered intraperitoneally every $60 \mathrm{~min}$, while continuously checking throughout the experiment the adequate level of anesthesia by means of the absence of the noxious hindpaw reflex. Staining of the diaphragmatic lymphatic was performed in vivo by an intraperitoneal injection of $0.8 \mathrm{ml}$ of $2 \%$ FITC-dextran in saline solution (Sigma-Aldrich) plus 10\% FITCfluorescent $0.1 \mu \mathrm{m}$ microspheres (Invitrogen). Because of their size, FITC-dextrans and microspheres cannot leave the peritoneal cavity directly into the bloodstream, but they do enter the lymphatic stomata of the peritoneal diaphragmatic mesothelium, thus efficiently loading the diaphragmatic lymphatic network. After $60 \mathrm{~min}$ from dye injection, the animals were turned supine, tracheotomized, paralyzed with a $0.3 \mathrm{ml}$ bolus of $2 \mathrm{mg} / \mathrm{ml}$ pancuronium bromide in saline solution in the jugular vein, and mechanically ventilated (Inspira, Harvard Apparatus) at a tidal volume and frequency set on the basis of the body weight. Then the chest wall was opened, the pleural diaphragmatic surface was exposed, and the superficial diaphragmatic lymphatic network containing the fluorescent dye could be visualized through a stereomicroscope in fluorescence epi-illumination (excitation $490 \mathrm{~nm}$, emission $520 \mathrm{~nm}$ ). Images of the peripheral diaphragmatic lymphatics, often arranged in loops, were video-recorded over time at $1-20 \mathrm{~Hz}$ with a cooled charge-coupled device camera (Orca ER, Hamamatsu) 
connected to a personal computer running SimplePCI software (Hamamatsu). At the end of the experiments, rats were euthanized with an anesthesia overdose.

This experimental protocol involving rats had been carefully reviewed and approved by the Ethical Committee of the University of Insubria.

Whole-mount staining and image acquisition. Immediately after the suppression of the animals at the end of experiments, the diaphragmatic tissue surrounding the same recorded vessels was fixed in situ with $4 \%$ paraformaldehyde for $10 \mathrm{~min}$, then excised and postfixed with $4 \%$ paraformaldehyde for $30 \mathrm{~min}$ and washed in PBS three times. Thereafter, specimens were permeabilized with $100 \%$ cold methanol and subsequently with $0.5 \%$ Triton X-100 and then incubated with blocking solution (1\% BSA, 5\% goat serum in PBS) for $1 \mathrm{~h}$ at room temperature. Overnight incubation with primary mouse anti $\alpha$-smooth muscle actin antibody [1:25 Actin Alpha 2 Smooth Muscle Antibody (1A4), Novus Biologicals] at $4^{\circ} \mathrm{C}$ followed by three washes in PBS and a 1-h incubation with a secondary goat anti-mouse antibody conjugated to Alexa Fluor 647 (1:100 goat anti-mouse IgG2a, Molecular Probes) was performed. Then specimens were rinsed three times in PBS, mounted onto glass coverslips with Fluoroshield (F6057, Sigma Aldrich), and imaged in their whole thickness with a confocal microscope (TCS SP5, Leica) with a $0.5-\mu \mathrm{m}$-axis steps: images were collected using a $\times 20$ dry objective and $98.33-\mu \mathrm{m}$ pinhole. The negative controls for the experiments were prepared and analyzed via similar procedures, omitting the incubation with the primary antibody; in addition, aorta specimens were used as positive controls. Negative and positive controls were scanned with the same instrument settings as the specimens for a valid comparison of relative fluorescence intensities.

Data analysis. The diameter of the observed lymphatic vessels was measured over time using "diameter" plug-in of ImageJ software (5). Briefly, SimplePCI proprietary video format was converted into TIFFbased image time-stacks, which were stabilized with built-in ImageJ plug-in. Then, to encompass the whole vessel diameter during the entire time stack, despite residual lateral movements of the vessel, the maximum intensity projection of time stack was used as a template to correctly position the diameter-measuring line perpendicularly to the vessel major axis. The analysis of the vessel diameter changes obtained over time from ImageJ was performed with Clampfit 10.2 (Molecular Devices); statistical analysis was performed using Origin software.

Analysis of the similarity of the vessel diameter over time signals as a function of a time-lag applied to one of them (cross-correlation analysis) was performed using Clampfit software. Confocal microscopy images were analyzed using Image J (National Institutes of Health) and Adobe Photoshop software. Pleural diaphragmatic lymphatic network overviews were obtained in silico by aligning and superimposing consecutive images into a continuous panorama using Adobe Photoshop software. Lymphatics cross sections were obtained using Re-slice function of Image $\mathbf{J}$ software on the merged $z$-stacks.

Data are presented as means $\pm \mathrm{SE}$. Difference between means were statistically evaluated with Student's $t$-test after data normality distribution check.

\section{RESULTS}

In the baseline period, after induction of deep anesthesia and throughout the entire experiments, mean arterial pressure and heart contraction frequency were $122 \pm 7 \mathrm{mmHg}$ and $118 \pm 9$ beats/min, respectively.

In vivo visualization of lymphatic structures and active pumping sites. A typical lymphatic arrangement of a portion of diaphragmatic network, as it appears under epifluorescent illumination when FITC-dextrans are injected into the peritoneal cavity, is presented in Fig. $1 B$. In this low-magnification image, which encompasses almost the entire right hemidiaphragm (as schematically shown in Fig. 1A), it is possible to recognize a network of vessels organized in linear tracts (arrows) connecting loop structures. The latter ones are present both on the medial muscular part of the diaphragm (at the center of the

Fig. 1. A: schematic drawing of the pleural surface of the diaphragm. The squared lines highlight the laterodorsal region of the right hemidiaphragm from where the in vivo fluorescent image of the diaphragmatic lymphatic network shown in $B$ was taken. $B$ : in vivo image of the diaphragmatic lymphatic network highlighted by an intraperitoneal injection of FITC-dextrans. The dotted line highlights the peripheral margin of the diaphragm. Lymphatic loops are preferentially located on the periphery of the diaphragm, while linear vessels emerge from them pointing toward the medial diaphragm surface (left side of the image). The box surrounds contracting sites of the upper loop, whose change in diameter over time can be appreciated in insets $a$ and $b$, reporting to two different images taken from a $1-\mathrm{Hz}$ frame rate video. $a$ : Loop segment in the diastolic phase. $b$ : Same site as in $a$ in the systolic phase. Scale bar is $1 \mathrm{~mm}$. Insets have the same magnification as the main panel.

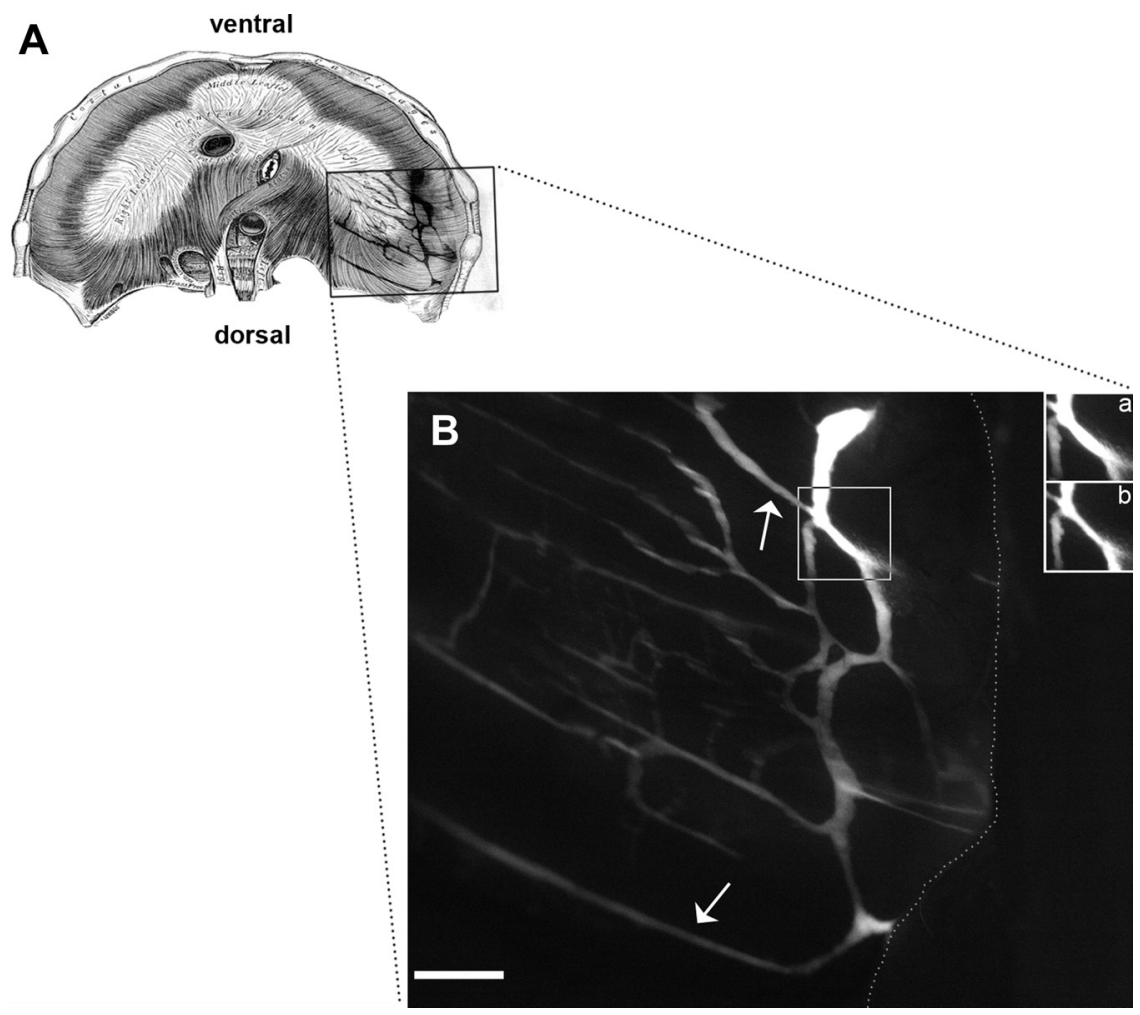


image), and at its far periphery toward the outer diaphragmatic margin (dotted line). Unlike what was observed in linear vessel of the medial portion of the network, the most peripheral loops are endowed with sites able to rhythmically change their diameter over time, as appreciable from the still images of insets $a$ and $b$, taken from a video recorded at $1-\mathrm{Hz}$ frame rate and showing a clear spontaneous reduction of vessel diameter over time.

Localization of smooth muscle around contracting and notcontracting sites. To investigate the organization of contractile elements in the wall of the peripheral loop vessels, we performed whole-mount immunostaining experiments against smooth muscle $\alpha$-actin on the very same loops that had been previously analyzed in vivo. In Fig. $2 A$, which presents a reconstruction of a whole peripheral loop from confocal images of several adjacent fields, the red and green signals highlight smooth muscle $\alpha$-actin in the vessel wall and FITC-dextran inside the vessels lumen, respectively. The images clearly show the presence of smooth muscle $\alpha$-actin in the vessel wall, although its distribution dramatically varies among different sites of the same loop. For example, in the segment illustrated in Fig. $2 A$, box $B^{\prime}$, smooth muscle is organized in a dense mesh of fibers which ensheath most of the circumference of the vessel, as clearly visible in the cross-section image show in Fig. $2 C$, taken at the white line $\left(C^{\prime}\right)$ of $2 B$. The capability of the vessel segment visualized in Fig. $2 B$ to actively contract was documented by the still images shown in $D$ and $E$, taken from the video recorded from the same site during the "in vivo" experiment, where the reduction of vessel diameter from the diastolic $(D)$ to the systolic $(E)$ phase over a 3-s time frame can be clearly appreciated. In other sites of the same loop
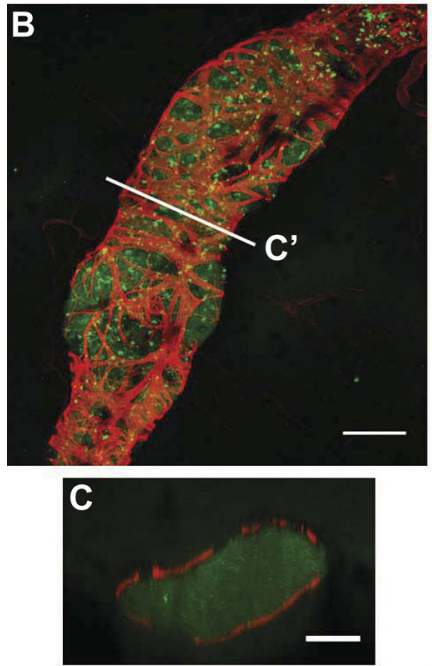

D
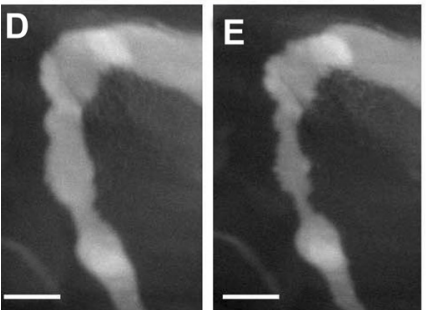
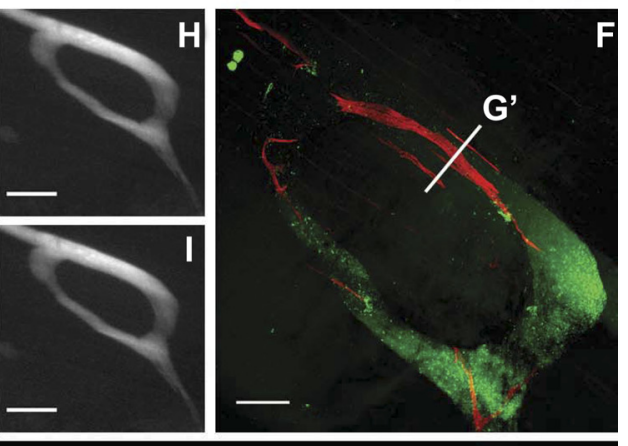

A
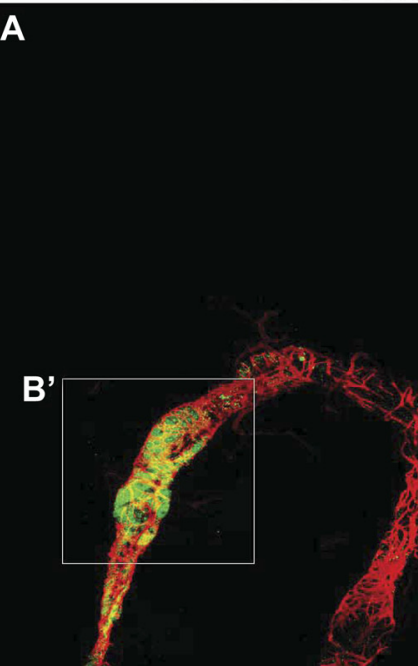

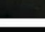
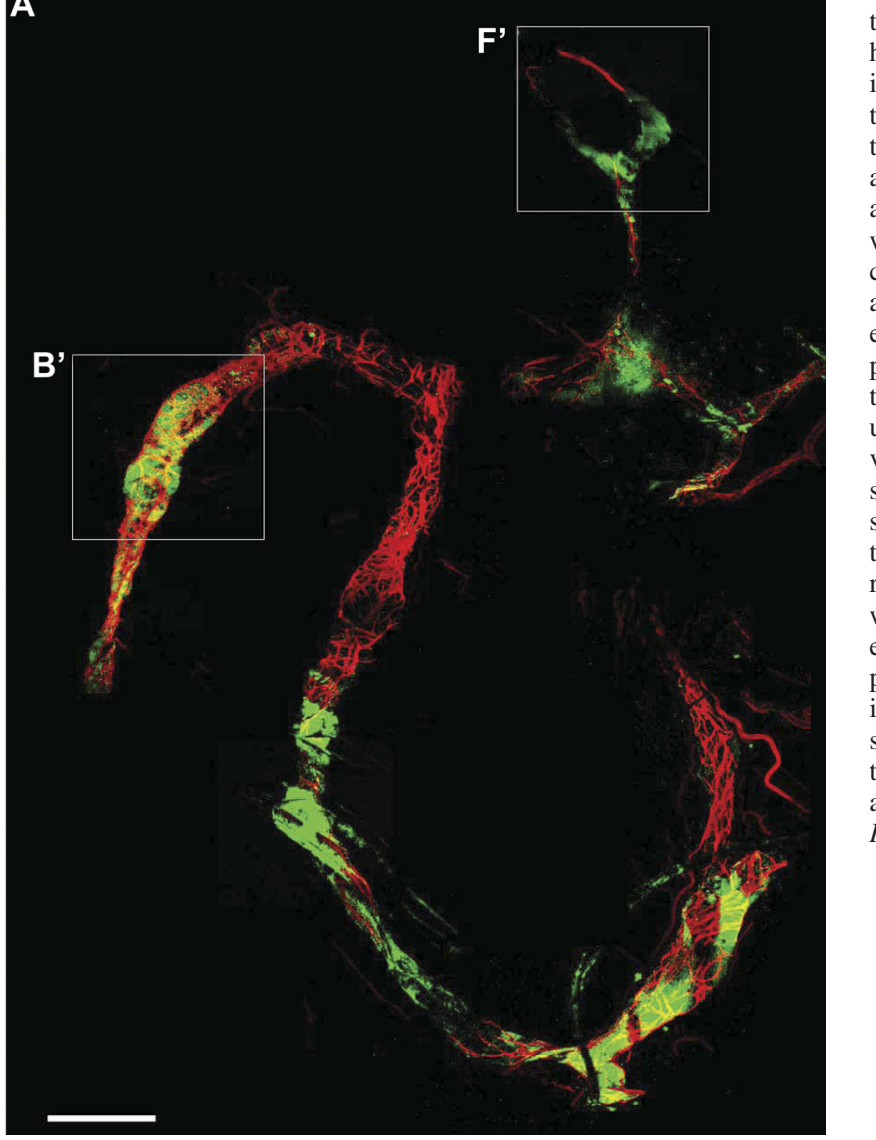

Fig. 2. Confocal whole-mount reconstruction of a previously in vivo imaged lymphatic loop. A: reconstruction of the whole lymphatic loop from confocal images taken at various sites. Red signal is anti-smooth muscle $\alpha$-actin; green signal is the residual FITC-dextran filling in the lymphatic vessels lumen. $B$ : higher magnification image from the site surrounded by the box $B^{\prime}$ in $A$, which had shown spontaneous contracting activity in the previously recorded video. $C$ : cross section reconstructed by $z$-series optical slices at the white line position $C^{\prime}$ in $B$ showing the almost complete disposition of smooth muscle around the whole vessel lumen, still filled with green FITC-dextran. Resting $(D)$ and contracting $(E)$ segments are of the same site as in $B$ and $C$, taken from two still images extracted from the video recording. In $E$, it is possible to appreciate the presence of "haustra" during contraction due to the discontinuous layer of smooth muscle surrounding the vessel. $F$ : higher magnification image from the site surrounded by the box $F^{\prime}$ in $A$, which had shown no spontaneous contracting activity in the previously recorded video. $G$ : cross section reconstructed by $z$-series optical slices at the white line position $\mathrm{G}^{\prime}$ in $F$ showing the presence of smooth muscle limited to very few points around the vessel wall. $H$ and $I$ : still images extracted from the video recording, showing no change in diameter over time at this site. Scale bars: $500 \mu \mathrm{m}(A), 100 \mu \mathrm{m}(B$ and $F), 50 \mu \mathrm{m}(C), 25 \mu \mathrm{m}(G), 250 \mu \mathrm{m}(D, E$, $H$, and $I$ ). 
Table 1. Functional values of the four classes of sites encountered in peripheral lymphatic loop

\begin{tabular}{|c|c|c|c|c|c|c|c|}
\hline & $n$ & Resting Diameter, $\mu \mathrm{m}$ & Distending Volume, pl & Stroke Volume, $\mathrm{pl}$ & $\begin{array}{c}\text { Ejection } \\
\text { Fraction, \% }\end{array}$ & $\begin{array}{c}\text { Frequency, } \\
\text { beats/min }\end{array}$ & $\begin{array}{l}\text { Lymph Flux, } \\
\mathrm{pl} / \mathrm{min}\end{array}$ \\
\hline Spontaneously active & 102 & $162.1 \pm 5.6$ & & $34.9 \pm 3.0$ & $43 \pm 2$ & $6.9 \pm 0.4$ & $199.3 \pm 13.9$ \\
\hline Inert & 78 & $154.4 \pm 7.4$ & & & & & \\
\hline Stretch activated & 8 & $147.2 \pm 13.2$ & $28.7 \pm 6.7$ & $64.4 \pm 9.7$ & $70 \pm 3$ & $2.5 \pm 0.1$ & $152.6 \pm 20.5$ \\
\hline$P$ value & & NS & & $<0.01$ & $<0.01$ & $<0.01$ & NS \\
\hline
\end{tabular}

Values are means \pm SE; $n$, no. of lymphatic sites. Values have been calculated as described in the APPENDIX. Distending volume represents the change in volume above resting volume. NS, nonsignificant.

(Fig. $2 A$, box $F^{\prime}$ and enlarged panel $F$ ), smooth muscle $\alpha$-actin, when present, showed a more sparse distribution with a predominant strip alignment parallel to the major axis of the lymphatic vessel, with the muscular layer surrounding only partially the vessel perimeter $(G)$. In vivo, this site showed no active contractions, as appreciable by the absence of diameter change over time in the still images taken from the previously recorded video (Fig. 2, $H$ and $I$ ).

Classification of vessel sites based on their segmental behavior. Through the systematic analysis of diameter changes over time performed at 197 sites from 15 loop structures recorded from 11 rats, it has been possible to identify, in vessel with similar diameter (Table 1) and even within the same loop (Fig. 3), four different types of segmental behavior. 1) In $51.8 \%$ of the observed lymphatic sites, active spontaneous contraction occurred at a mean frequency of $6.9 \pm 0.4$ beats $/ \mathrm{min}(n=102)$. In these vessels (spontaneously active, Fig. 3A), the average diastolic diameter $(162.1 \pm 5.6 \mu \mathrm{m}, n=102)$ sharply and significantly $(P<0.01$, paired $t$-test, $n=102)$ decreased by $74.5 \pm 1.2 \%$ of the diastolic value in the systolic phase, thereby returning to the precontractile diameter with a slower kinetics. 2) In $39.6 \%$ of the lymphatic (inert) segments, the diameter $(154.4 \pm 7.4 \mu \mathrm{m}, n=78)$ never changed over the observation period, extended up to $90 \mathrm{~s}$ (Fig. 3B). 3) Approximately $4.5 \%$ of the vessel segments displayed a pure passive behavior (passive sites, Fig. 3C). From its baseline value $(185.9 \pm 32.2 \mu \mathrm{m}, n=9)$, vessel diameter periodically $(2.9 \pm$ 0.5 beats $/ \mathrm{min}$ ) sharply enlarged to $133.63 \pm 7.32 \%$ of its resting value, thereby slowly decaying to the baseline diameter. 4) The remaining $4.1 \%$ of the lymphatic sites displayed a biphasic behavior (Fig. 3D) at an average rate of $2.5 \pm 0.1$ beats/min. Starting from a resting value of $147.2 \pm 13.2 \mu \mathrm{m}$ $(n=8)$, the diameter first enlarged up to $123.8 \pm 7.6 \%$ of the resting value $(P<0.01, n=8$, paired $t$-test $)$ and then dropped to $66 \pm 1.6 \%(P<0.01, n=8$, paired $t$-test $)$. At variance with what was observed for purely passive sites of Fig. $3 C$, at this sites a passive enlargement of the vessel triggered an active, stretch-activated response. No significant differences $(P=$ 0.448, one-way ANOVA, 196 degrees of freedom) in resting diameter was encountered among the four populations of lymphatic vessel segments.

Temporal correlation analysis among different sites of the same lymphatic loop. The distribution of the four types of lymphatic segments within a whole lymphatic loop was very variable. When multiple spontaneously active sites were scattered within a sequence of inert sites, the former displayed their typical contractile frequency ( $\sim 7$ beats/min). Instead, if stretch-activated and purely passive sites were encountered in proximity of spontaneously active sites, they all rhythmically contracted at a lower frequency $(\sim 2-3$ beats $/ \mathrm{min})$. In the lymphatic network of the most peripheral muscular diaphragm, multiple spontaneously active sites can be encountered in the same loop, as in the example of Fig. $4 A$, where, as appreciable in $4 C$, spontaneous contraction were observed at sites 1,2 , and 3 , while site 4 was purely passive. Sites 2 and 3 displayed a closely correlated auto-rhythmic activity with a contraction frequency of 3.1 beats/min and a site 2 to 3 time lag of $0.5 \mathrm{~s}$; passive site 4 displayed the same frequency of enlargement, with a site 3 to 4 delay of $0.95 \mathrm{~s}$. The spontaneous contractile frequency ( 5.4 beats/min) of site 1 was instead completely uncorrelated with the other sites. Nevertheless, all active sites contributed to the ordered flux of lymph in the loop structure, as witnessed by the progressive movement of a fluorescent aggregate inside the vessel lumen (Fig. 4B, arrow). As evident from Fig. 5, $B$ and $C$, a spontaneous contraction is always the first event observable in a given loop (solid line, trace 1),
Fig. 3. Different mechanical behavior of lymphatic segments belonging to the same lymphatic loop. White arrowheads identify the sites whose diameter change over time is reported in the four panels surrounding the image. Scale bar $1 \mathrm{~mm}$. A: spontaneously active site, exhibiting spontaneous contractions as witnessed by the downward deflections of the diameter trace. $B$ : inert site, where vessel diameter never changes over time, even if the segment is in close proximity and functionally connected to nearby active sites. $C$ : passive site showing a passive increase in diameter followed by slow return to baseline resting value. $D$ : stretch-activated site, displaying an initial increase in diameter (upward trace deflection), immediately followed by an active contraction (downward deflection).

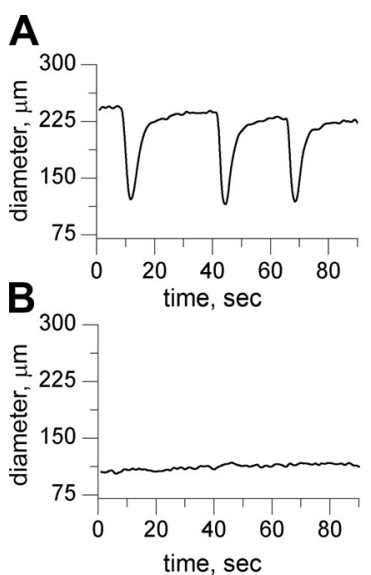

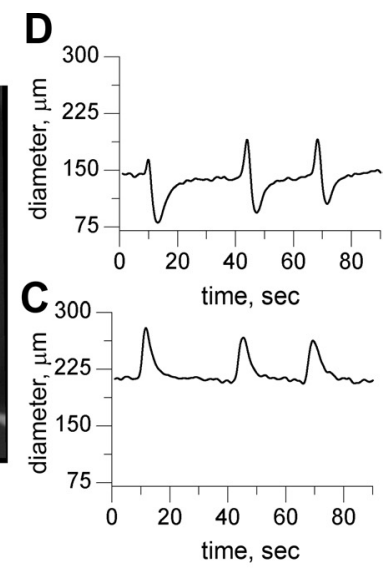


A

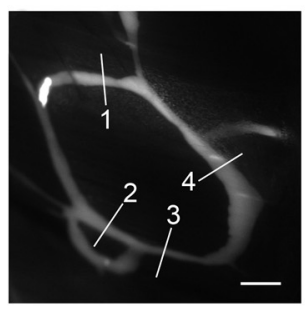

B
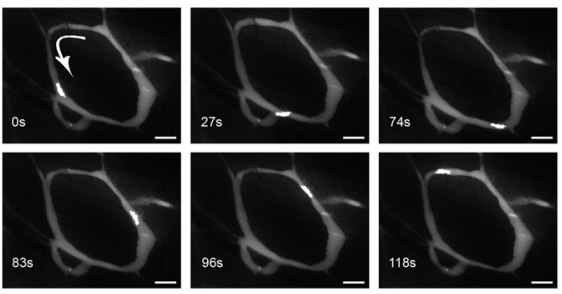

C

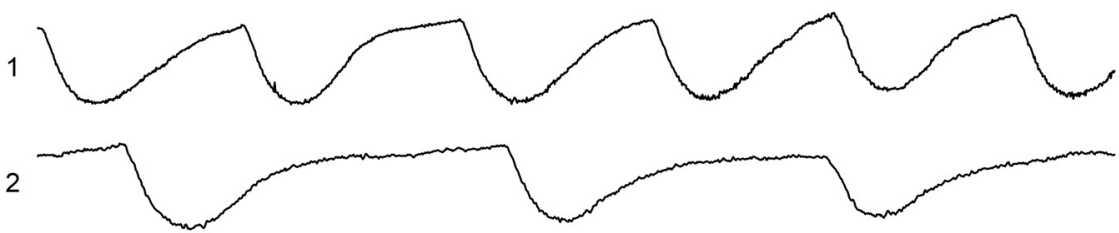

3

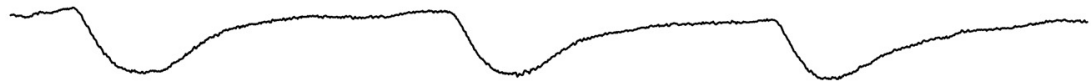

4

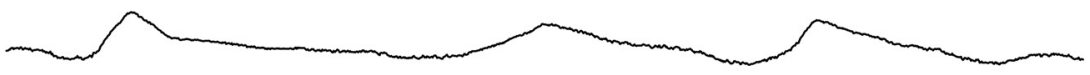

$50 \mu \mathrm{m}$

$10 \mathrm{sec}$
Fig. 4. Temporal correlation among functionally different segments of the same lymphatic loop. A: fluorescence image of a FITC-dextran filled lymphatic loop showing a fluorescence aggregate (top left bright object inside the lumen) circulating inside the loop. Sites 1-4 indicate where the corresponding traces of $C$ have been measured. Scale bar $500 \mu \mathrm{m}$. $B$ : subsequent still images taken from the video recording, showing the intraluminar progression of the fluorescent aggregate in a counterclockwise direction, as indicated by the arrow. Scale bar $500 \mu \mathrm{m}$. $C$ : plots of diameter change over time for sites 1-4 indicated in A. Downward trace deflection indicates reduction in vessel diameter (contraction); upward trace deflection indicates increase in vessel diameter (expansion). Although sites 1-3 are all actively contracting, they display quite at different paces. Site 4 passively enlarges at a frequency dictated by sites $2-3$. followed by the stretch-activated segments (dark shaded line, trace 2 ) and eventually by passive segments (light shaded line, trace 3$)$. The time delay $(\tau, \mathrm{s})$ between the diameter change in spontaneously active and stretch-activated or passive segment was, on average, $0.35 \pm 0.1 \mathrm{~s}$ and $0.54 \pm 0.1 \mathrm{~s}$, respectively $(n=4)$, and was related to the distance $(d, \mu \mathrm{m})$ between the spontaneously active and the stretch-activated or passive segment, as described by the linear relationship: $\tau=0.0002+$ $0.063 d,\left(R^{2}=0.67, n=12\right)$. To better investigate this phenomenon in loops characterized by multiple spontaneously contractile sites, the mean percent diameter changes were plotted as a function of the corresponding mean contraction frequency. Data could be fitted by a rectangular hyperbola (Fig. 6) of the kind:

$$
y=y_{0}+\frac{a \cdot b}{b+x}
$$

Where $y_{0}=3.94, a=71.92, b=2.27, R^{2}=8.84, P<0.01$.

Being the $(x \cdot y)$ product, i.e., the (contractile frequency $\Delta$ diameter) $=$ flow index constant in the hyperbolic functions, this result mathematically confirms the visual observation that the average intraluminar flow is nearly constant within a loop, despite spontaneous variable contractile rates at various segmental sites.

Examining the diameter over time signals measured in loops in which at least two spontaneously active sites contracted at the same frequency but with a finite time delay, we performed a cross-correlation analysis to calculate the spreading velocity of the contraction wave along the lymphatic vessel wall. By dividing the distance along the vessel between neighboring contracting sites by the time lag of their contractions, the mean contractile wave spreading velocity was $1.3 \pm 0.3 \mathrm{~mm} / \mathrm{s}(n=$ 5 loops).

\section{DISCUSSION}

Spontaneously contracting diaphragmatic lymphatic segments. The present study reveals for the first time the coexistence of actively pumping, stretch-dependent pumping, and completely passive sites within the diaphragmatic lymphatic network. The functional difference between various sites, encountered even within the same lymphatic loop, never emerged in the past. Indeed, the greatest majority of the available intraluminar diaphragmatic lymphatic pressure $\left(\mathrm{P}_{\text {lymph }}\right)$ recordings was characterized by pressure swings, which could be directly related to the mechanical impact of the cardiac (17) or respiratory (11) activity on the diaphragmatic tissue and on lymphatic vessels. Being spontaneous $\mathrm{P}_{\text {lymph }}$ swings extremely rare events (14), we inferred that diaphragmatic lymphatic function was almost exclusively sustained and modulated by tissue movements and by the mechanical properties of the tissue $(9,16)$. This view was supported by morphological evidences showing that lymphatics located in the submesothelial interstitium on both the pleural and peritoneal sides and among the muscle fibers of the diaphragm essentially lack smooth muscle cells (7). However, $\mathrm{P}_{\text {lymph }}$ measurements had always been obtained from superficial submesothelial lymphatic vessels, running over the pleural side of the muscular lateral diaphragmatic dome, which is the most easily accessible, both for stereomicroscopic observation and for micropuncture approach. Indeed, submesothelial vessels had been visualized through pleural injections of fluorescent dextrans, which are drained first into the subpleural superficial lymphatic network with a very minor filling of the deeper diaphragmatic lymphatics $(10,17)$.

In the present work, a different portion of the diaphragmatic lymphatic network, located at the extreme diaphragmatic margin in close proximity with the abdominal wall, has been studied. Lymphatic vessels of this area could be readily and 
A

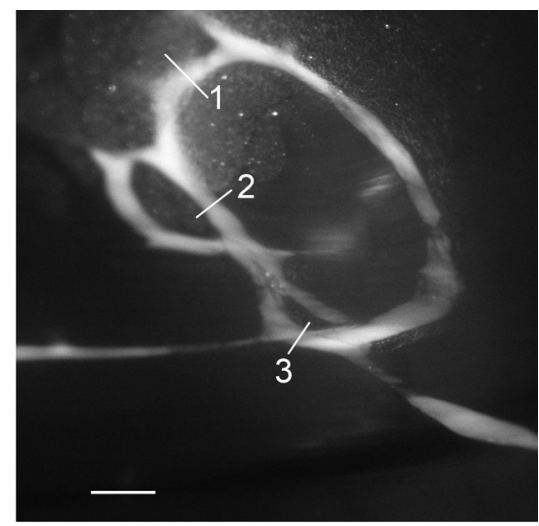

B

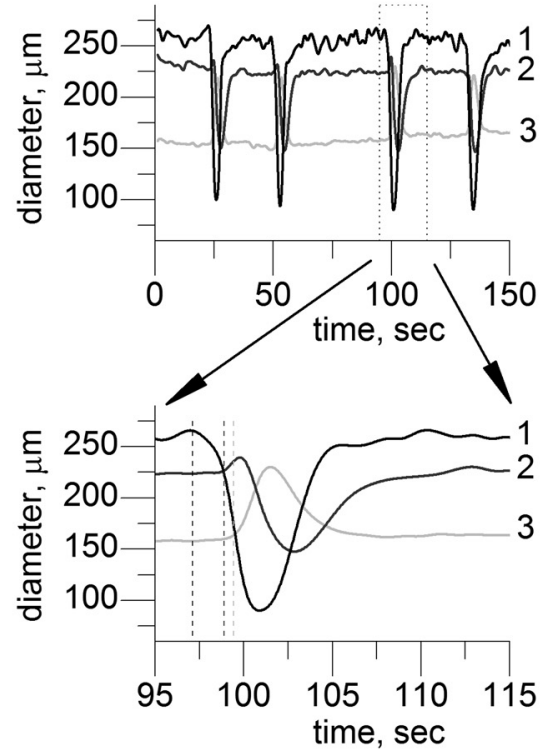

Fig. 5. Sequential activation of different sites in the same lymphatic loop. $A$ : fluorescence image of a FITC-dextran filled lymphatic loop. Lines identify the position of sites 1-3 where the corresponding traces of $B$ and $C$ have been measured. Scale bar $500 \mu \mathrm{m}$. B: time-aligned traces of diameter change of spontaneously active (solid line; 1 ), stretch-activated (dark shaded line; 2 ), and passive (light shaded line; 3 ) sites recorded from the same loop over a 150-s period. $C$ : enlarged view of the area boxed in $B$. The dotted vertical lines identify in temporal sequence: in trace 1, the beginning of spontaneous activation; in trace 2, the beginning of passive distension triggering the stretch-activated contraction; in trace 3, the onset of passive distension.

reproducibly stained by injections of FITC-conjugated dextrans in the peritoneal cavity (Fig. 1), a procedure that not only eliminates the fluorescent halo usually observed on the pleural surface of the diaphragm after intrapleural FITC injection, but, more importantly, fills the deepest lymphatic vessels, making them visible. Although observable from the pleural diaphragmatic dome, the spontaneously contracting lymphatic loops investigated in the present study are located in the most peripheral margin of the peritoneal diaphragm, likely in close proximity to lymphatic vessels belonging to the peritoneal wall. Indeed, it has been shown (10) that, at $30 \mathrm{~min}$ from intraperitoneal injection, more than $90 \%$ of the injected fluorescent dextrans accumulated in the transverse lymphatic ducts proximal to peritoneal surface and in the deeper central collectors, with only less than $1 \%$ of the tracer staining the submesothelial lacunae opposite the site of injection. Because of their depth within the diaphragmatic tissue and their extremely peripheral location, so far it has not been possible to attain direct $\mathrm{P}_{\text {lymph }}$ measurements in these vessels.

Active contractions rely on the presence of smooth muscle cells in the wall of lymphatic vessels. However, at least in the diaphragmatic lymphatic vessels, the presence of the smooth muscle alone is not an indication of a spontaneous contractile behavior per se. Indeed, although both sites of Fig. 2, $B$ and $F$, do possess smooth muscle fibers in their respective walls, only the former displayed a spontaneous contractile activity, while site of Fig. $2 F$ belongs to the passive type. The difference between spontaneously contracting vessels and completely passive or inert vessel segments seems to depend on smooth muscle organization rather than on its presence alone. Indeed, in the former sites, smooth muscle fibers are organized in a dense net surrounding the whole circumference of the vessel (Fig. 2C); instead, passive or inert sites either do not possess smooth muscle fibers, or, if they do, they are sparse, preferentially aligned along the major axis of the vessel, and do not encircle the whole vessel wall (Fig. $2 G$ ), a disposition that does not appear to be finalized to attain an efficient vessel constriction. Longitudinal smooth muscle fibers might, in principle, cause shortening of the vessels. However, the outer surface of the diaphragmatic lymphatics appears to be tightened to the surrounding extracellular matrix fibrils, to skeletal muscle, and, in case of more superficial vessels, to the parietal mesothelium (7); hence, they do not seem capable of significant shortening, and indeed we were not able to record any shortening of lymphatic segments along their major axis. If compared with the compact spindle-shaped organization of smooth muscle in arterioles (13), it is clear that smooth muscle around contracting lymphatic vessels is less strictly organized. This difference may be explained considering that, while the arteriolar smooth muscle has to contract against an intraluminal hydraulic pressure of $\sim 80 \mathrm{mmHg}$, baseline $\mathrm{P}_{\text {lymph }}$ in diaphragmatic lymphatic vessels may vary between -10 and $8-9 \operatorname{mmHg}(9,11$, 17). Spontaneous contraction implies the existence, within the population of smooth muscle cells surrounding the vessel wall, of a subset of pacemaking cells (20-22) triggering the contractile wave. However, based on images such as those presented in Fig. 2, it was not possible to identify the pacemaking from the classical not pacemaking smooth muscle cells.

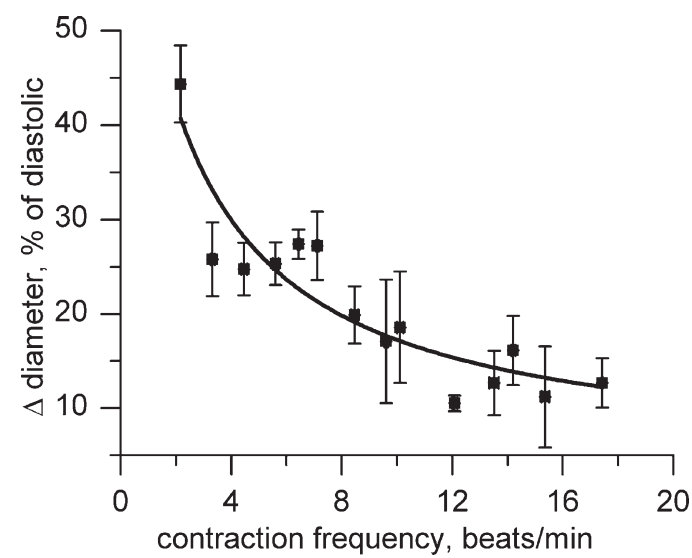

Fig. 6. Hyperbolic fit of the relationship between contraction frequency (beats/min) and diameter systolic change $(\Delta$, percentage of diastolic diameter) in spontaneously contracting vessels belonging to loop structures. 
The presence of both circular and longitudinal smooth muscle in the vessel wall may also be relevant from the mechanical standpoint. Indeed it has been shown (9) that the presence of muscular component around the lymphatic vessel may decrease by up to two orders of magnitude the mechanical compliance of the wall, allowing a better transmission of the external tissue forces to the vessel lumen. The spontaneously contractile vessels investigated in the present study are located in the extreme periphery of the diaphragm, where tissue displacements depending on the respiratory and the cardiac activity might be reduced compared with more central regions of the diaphragm: hence, a smooth muscle support might serve at maintaining lymphatic function in vessels otherwise poorly assisted by the extrinsic mechanism.

Indications on the actual role played by the various segments' typology may be derived by analyzing their functional parameters (Table 1). As derived from traces of Figs. 3 and 5, both stretch-activated and passive segments receive, during their diastolic phase, a fluid volume, defined distending volume (Table 1), that increases the diameter of the vessel above their end-diastolic value. The distending volume is nil in actively contracting vessels in which the vessel returns to its presystolic value at the end of the diastole. As described in the APPENDIX, stroke volume is defined as the difference between distending and end-systolic volume and can be thus calculated only for spontaneously contracting and stretch-activated segments. By assuming an elliptical cross-sectional area of the diaphragmatic lymphatic vessels (8) with a 1:0.35 ratio between the major diameter (parallel to the pleural diaphragmatic surface and directly measured during video recording) and the minor diameter (perpendicular to the diaphragmatic surface), it has been possible to calculate (APPENDIX) the following: 1) the distending volume (for purely passive and stretch-activated vessels); 2) the stroke volume and the ejection fraction (in spontaneously contracting and stretch-activated segments); and 3 ) the lymph flux in all ideal vessel segments purposely set 10 $\mu \mathrm{m}$ long (Table 1). After distension, passive vessels discharge $\sim 40 \%$ of the accumulated volume. Vice versa, during diastole, spontaneously contracting vessels do not distend above their resting volume, suggesting that their smooth muscle wall is compliant at the beginning of the diastole and becomes progressively stiffer when approaching its diastolic resting value, thus preventing further vessel enlargement. Hence, spontaneously contracting vessels seem to play an exclusively propelling function, which might, on one hand, organize the intraluminar flow direction and, on the other, by maintaining a high muscular tone, amplify the intraluminar pressure swing caused by external tissue movements. It is interesting to note that the ejection fraction of diaphragmatic spontaneously active segments $(\sim 43 \%$, Table 1$)$ does not greatly differ from that recorded in mesenteric lymphatics ( $\sim 67 \%$; Ref. 4$)$; this suggests that the intrinsic contraction properties tend to be preserved in the face of a very different contribution of the extrinsic pumping mechanism, which is most prominent in diaphragmatic, but scant if any in mesenteric, lymphatics.

Stretch-activated and passive lymphatic segments. Data of Table 1 highlight the functional differences between the passive and the stretch-activated vessel segments. The large distending volume encountered in purely passive vessels indicates that, for a given vessel diameter, they are also the most compliant ones, better fit to accommodate a large amount of fluid, thus acting as a passive fluid reservoir of lymph absorbed from the surrounding interstitial space and/or pleural or peritoneal cavity. These segments may also represent lymph buffer sites, where the intraluminal volume increase limits the hydraulic pressure buildup, but it is not to be excluded that they may also represent, in line with the windkessel effect (6), sites where the elastic recoil of the lymphatic vessel wall turns a pulsatile flux continuous, thus promoting a larger overall lymph flux down the lymphatic vessel system, in accordance with what happens in arteries.

Stretch-activated segments may instead represent sites where fluid absorption is coupled to propulsion or, mechanically speaking, where the distending pressure exerted on the lymphatic wall and the resulting increased wall tension triggers an active contraction of lymphatic smooth muscle cells to propel the lymph. Indeed, to be able to contract, these vessels need to collect fluid, either absorbed of flowing from another vessel segment, to increase their volume above the resting value (Table 1). The temporal delay between changes in diameter of stretch-activated and passive segments (Fig. 5C, traces 2 and 3, respectively) indicates that, within a lymphatic loop, the pace set by stretch-activated segments imposes the filling/emptying rate of passive vessels. This suggests that 1 ) stretch-activated and passive segments lay in functional proximity within the loop; and 2) within the loop the fluid is propelled by the stretch-activated to the passive segments, as suggested also by the identity between the stroke volume of the former and the distending volume of the latter (Table 1). The significantly higher contraction frequency observed in spontaneously active segments, whose wall presents a rather homogenous distribution of actin, compared with stretch-activated and passive segments, characterized by scanty and/or longitudinal actin distribution, does not seem to correlate with segment resting diameter, which was similar in all observed segments.

A last remark is due on the inert segments, which seem to be completely extraneous to the loop fluid dynamics. These sites might correspond to points of confluence between segments of the superficial loops and vessels departing perpendicularly to the loop to reach the lymphatic collectors in the center of the diaphragmatic thickness (7); the fluid arriving to these branching points would leave the superficial circuit to move to central diaphragmatic collectors without affecting the diameter of the observed loops.

Temporal and functional coordination in lymphatic loops. Actively contracting sites were found, within the same loop, in proximity of not contracting or passive sites. On the other hand, the latter ones were never present alone, but always in proximity of active and stretch-dependent sites. The analysis of diameter changes over time (Fig. $4 C$ ) of different segments belonging to the same loop clearly indicates that they are arranged into functional units distinguishable on the basis of their contraction frequency and spontaneously active, stretchactivated, or passive behavior. Cross-correlation analysis showed that, when all vessel typologies were present in a loop, the change in diameter wave was first triggered at a spontaneously contracting site to be subsequently propagated to the stretchactivated segments and finally to the passive ones (Fig. 5). Such a temporal sequence of events is in very close agreement with the fact that the stroke volume of spontaneously active segments (Table 1) is similar to stretch-activated distending 
volume, while stretch-activated stroke volume is consistent with distending volume in purely passive sites. As expected, the time delay was correlated to the distance between actively contracting and stretch activated or passive segments. However, since only $\sim 70 \%$ of the time delay variability was accounted for by distance, other components, like for example the local compliance of the vessel sites, may play a role in delaying the wave. The propagation velocity of the contraction wave along different loops in the diaphragm (about $1.3 \mathrm{~mm} / \mathrm{s}$, see RESULTS) is lower if compared with that measured in peritoneal lymphatic vessels $(4-8 \mathrm{~mm} / \mathrm{s}$, Refs. 2, 23), albeit the contraction frequency of $\sim 7$ beats/min found in the present study is in line with that reported for the same vessels in the peritoneal cavity [ $\sim 9$ beats/min in age-matched rats (1)]. Thus it is conceivable that the electric conduction pathway of smooth muscle cells belonging to diaphragmatic lymphatic loops may differ in respect to linear peritoneal lymphatics. However, given that the linear diaphragmatic lymphatics, located medially on muscular and tendineous portion of the pleural diaphragmatic dome, do not seem to possess an intrinsic spontaneous activity, it remains to be resolved if the major source of difference in the propagation velocity of the contraction wave resides in the linear or in the loop type of vessel rather than in their tissue localization.

Albeit variable, the heterogeneous combination of sites is, however, functionally coordinated in that loops are able to sustain a unidirectional and constant lymph flux, as visually indicated by the one-way movement of fluorescent aggregate (Fig. $4 B$ ) and postulated by a mathematical model of a lymphatic vessel segment composed of several lymphangions (19). In particular, sites with a lower contraction frequency display a larger stroke volume and vice versa (Table 1, Fig. 6). The lymph flow in these deep loops at the extreme peripheral edge of the diaphragm is much smaller compared with what previously derived from direct measurement of flow velocity (8) in loops $(6.9 \mu \mathrm{l} / \mathrm{min})$ and linear vessels $(10.6 \mu \mathrm{l} / \mathrm{min})$ running over the muscular and medial tendineous pleural diaphragmatic dome, respectively. Although identification of the sites where lymph leaves the diaphragm is beyond the aims of the present study, the progressive increase in segmental lymph flow from the peripheral to the medial diaphragm suggests that lymph drained from the pleural and peritoneal cavities and from the diaphragmatic interstitium is conveyed first to the loops at the sites of lymph formation to be subsequently centripetally emptied into fewer and larger linear lymphatics running through the central diaphragmatic tendon.

Conclusion. Overall, our data show that, unlike most of the other diaphragmatic lymphatics, those supplying the most peripheral peritoneal side of the diaphragm exploit active contractions to propel lymph toward the rest of the diaphragmatic lymphatic network. At the extreme periphery of the diaphragm, these lymphatic loops compensate for the lack of strong tissue motion, which support most of lymph flux within the medial diaphragmatic lymphatic network, through a well-coordinated functional synergy between spontaneously contracting, stretchactivated, and passive vessel segments, thus guaranteeing fluid drainage from the pleural apposition zone and the perihepatic abdominal intraserosal fluid pools facing the lateral edges of the diaphragm.

\section{APPENDIX}

Lymphatic vessels functional parameters were estimated as follows:

1) Stroke volume (SV; in $\mathrm{pl})$ :

$$
\mathrm{SV}=\frac{\left[\left(r_{\mathrm{D}} \cdot 0.35 r_{\mathrm{D}} \cdot \pi\right)-\left(r_{\mathrm{S}} \cdot 0.35 r_{\mathrm{S}} \cdot \pi\right)\right] \cdot 10}{10^{3}}
$$

where $r_{\mathrm{D}}(\mu \mathrm{m})$ is diastolic (distention peak value for stretch-activated vessels) radius; $r_{\mathrm{S}}(\mu \mathrm{m})$ is systolic radius (for spontaneously active and stretch-activated segments); $10(\mu \mathrm{m})$ is lymphatic vessel tract in which SV was estimated; 0.35 is smaller transverse-to-the larger perpendicular lymphatic radius ratio; and $10^{3}$ is $\mu \mathrm{m}^{3} / \mathrm{pl}$ conversion factor.

2) Distending volume (DV; in pl):

$$
\mathrm{DV}=\frac{\left[\left(r_{\mathrm{E}} \cdot 0.35 r_{\mathrm{E}} \cdot \pi\right)-\left(r_{\mathrm{R}} \cdot 0.35 r_{\mathrm{R}} \cdot \pi\right)\right] \cdot 10}{10^{3}}
$$

where $r_{\mathrm{E}}(\mu \mathrm{m})$ is fully expanded radius; $r_{\mathrm{R}}(\mu \mathrm{m})$ is resting radius; and $10(\mu \mathrm{m})$ is lymphatic vessel tract in which DV was estimated.

3) Ejection fraction (EF; in \%):

$$
\begin{aligned}
& \mathrm{EF}_{\mathrm{S}}=\frac{\mathrm{SV}}{\mathrm{RV}} \\
& \mathrm{EF}_{\mathrm{D}}=\frac{\mathrm{DV}}{\mathrm{RV}}
\end{aligned}
$$

where $\mathrm{EF}_{\mathrm{S}}$ is spontaneously active and stretch-activated lymphatic vessel $\mathrm{EF} ; \mathrm{EF}_{\mathrm{D}}$ is passive lymphatics $\mathrm{EF}$; and $\mathrm{RV}$ is resting volume (pl), estimated as:

$$
\mathrm{RV}=\frac{\left(r_{\mathrm{D}} \cdot 0.35 r_{\mathrm{D}} \cdot \pi\right) \cdot 10}{10^{3}}
$$

where $10(\mu \mathrm{m})$ is lymphatic vessel tract in which DV was estimated.

4) Lymph flux (LF; in $\mathrm{pl} / \mathrm{min})$ :

$$
\mathrm{LF}=\mathrm{SV} \cdot \mathrm{CF}
$$

where $\mathrm{CF}$ is spontaneously active and stretch-activated contraction frequency (beats/min).

\section{ACKNOWLEDGMENTS}

Authors are thankful to Luisa Guidali for precious help with the acquisition of confocal images, and to Dr. Graziella Santoro for help in managing the laboratory work and data acquisition.

\section{GRANTS}

This work was supported by Fondo di Ricerca di Ateneo (FAR) 2011 and FAR 2012 to D. Negrini and A. Moriondo from the University of Insubria.

\section{DISCLOSURES}

No conflicts of interest, financial or otherwise, are declared by the author(s).

\section{AUTHOR CONTRIBUTIONS}

Author contributions: A.M. and D.N. conception and design of research; A.M., E.S., and C.M. performed experiments; A.M., E.S., and C.M. analyzed data; A.M. and D.N. interpreted results of experiments; A.M. prepared figures; A.M. drafted manuscript; A.M., E.S., C.M., and D.N. approved final version of manuscript; D.N. edited and revised manuscript.

\section{REFERENCES}

1. Akl TJ, Nagai T, Cote GL, Gashev AA. Mesenteric lymph flow in adult and aged rats. Am J Physiol Heart Circ Physiol 301: H1828-H1840, 2011. 
2. Akl TJ, Nepiyushchikh ZV, Gashev AA, Zawieja DC, Cot GL. Measuring contraction propagation and localizing pacemaker cells using high speed video microscopy. J Biomed Opt 16: 026016, 2011.

3. Azzali G. The lymphatic vessels and the so-called "lymphatic stomata" of the diaphragm: a morphologic ultrastructural and three-dimensional study. Microvasc Res 57: 30-43, 1999.

4. Benoit JN, Zawieja DC, Goodman AH, Granger HJ. Characterization of intact mesenteric lymphatic pump and its responsiveness to acute edemagenic stress. Am J Physiol Heart Circ Physiol 257: H2059-H2069, 1989.

5. Fischer MJ, Uchida S, Messlinger K. Measurement of meningeal blood vessel diameter in vivo with a plug-in for ImageJ. Microvasc Res 80: $258-266,2010$.

6. Frank $\mathbf{O}$. The basic shape of the arterial pulse. First treatise: mathematical analysis 1899. J Mol Cell Cardiol 22: 255-277, 1990.

7. Grimaldi A, Moriondo A, Sciacca L, Guidali ML, Tettamanti G, Negrini D. Functional arrangement of rat diaphragmatic initial lymphatic network. Am J Physiol Heart Circ Physiol 291: H876-H885, 2006.

8. Moriondo A, Bianchin F, Marcozzi C, Negrini D. Kinetics of fluid flux in the rat diaphragmatic submesothelial lymphatic lacunae. Am J Physiol Heart Circ Physiol 295: H1182-H1190, 2008.

9. Moriondo A, Boschetti F, Bianchin F, Lattanzio S, Marcozzi C, Negrini D. Tissue contribution to the mechanical features of diaphragmatic initial lymphatics. J Physiol 588: 3957-3969, 2010.

10. Moriondo A, Grimaldi A, Sciacca L, Guidali ML, Marcozzi C, Negrini D. Regional recruitment of rat diaphragmatic lymphatics in response to increased pleural or peritoneal fluid load. J Physiol 579: 835-847, 2007.

11. Moriondo A, Mukenge S, Negrini D. Transmural pressure in rat initial subpleural lymphatics during spontaneous or mechanical ventilation. Am J Physiol Heart Circ Physiol 289: H263-H269, 2005.

12. Muthuchamy M, Zawieja D. Molecular regulation of lymphatic contractility. Ann N Y Acad Sci 1131: 89-99, 2008.
13. Nees S, Juchem G, Eberhorn N, Thallmair M, Forch S, Knott M, Senftl A, Fischlein T, Reichart B, Weiss DR. Wall structures of myocardial precapillary arterioles and postcapillary venules reexamined and reconstructed in vitro for studies on barrier functions. Am J Physiol Heart Circ Physiol 302: H51-H68, 2012.

14. Negrini D, Del Fabbro M. Subatmospheric pressure in the rabbit pleural lymphatic network. J Physiol 520: 761-769, 1999.

15. Negrini D, Moriondo A. Lymphatic anatomy and biomechanics. $J$ Physiol 589: 2927-2934, 2011.

16. Negrini D, Moriondo A. Pleural function and lymphatics. Acta Physiol (Oxf) 207: 244-259, 2013.

17. Negrini D, Moriondo A, Mukenge S. Transmural pressure during cardiogenic oscillations in rodent diaphragmatic lymphatic vessels. Lymphat Res Biol 2: 69-81, 2004.

18. Ohtani O, Ohtani Y. Recent developments in morphology of lymphatic vessels and lymph nodes. Ann Vasc Dis 5: 145-150, 2012.

19. Venugopal AM, Stewart RH, Laine GA, Dongaonkar RM, Quick CM. Lymphangion coordination minimally affects mean flow in lymphatic vessels. Am J Physiol Heart Circ Physiol 293: H1183-H1189, 2007.

20. von der Weid PY. Review article: lymphatic vessel pumping and inflammation-the role of spontaneous constrictions and underlying electrical pacemaker potentials. Aliment Pharmacol Ther 15: 1115-1129, 2001.

21. von der Weid PY, Rahman M, Imtiaz MS, van Helden DF. Spontaneous transient depolarizations in lymphatic vessels of the guinea pig mesentery: pharmacology and implication for spontaneous contractility. Am J Physiol Heart Circ Physiol 295: H1989-H2000, 2008.

22. von der Weid PY, Zawieja DC. Lymphatic smooth muscle: the motor unit of lymph drainage. Int J Biochem Cell Biol 36: 1147-1153, 2004.

23. Zawieja DC, Davis KL, Schuster R, Hinds WM, Granger HJ. Distribution, propagation, and coordination of contractile activity in lymphatics. Am J Physiol Heart Circ Physiol 264: H1283-H1291, 1993. 